\title{
Subclavian Artery Versus Femoral Artery Cannulation as An Access for Cardiopulmonary Bypass in Repair of Acute Type A Aortic Dissection
}

\author{
Fouad M. Rasekh, Ahmed S. Mahmoud* \\ *Cardiothoracic Surgery, Faculty of Medicine, Cairo University \\ Corresponding author: Ahmed Sayed Hussein, E-mail: drahmed 755@gmail.com, Telephone: 00201090991111
}

\begin{abstract}
\section{INTRODUCTION}

Surgical of acute aortic dissection type A (ATAAD) is considered a big challenge to all cardiac surgeons. One of the great difficulties involved in this procedure is how to establish adequate extracorporeal circulation, dealing with the friable aortic wall tissues, and to ensure adequate cerebral protection especially during the deep hypothermic total circulatory arrest.

Over many years cardiac surgeons used to use femoral artery in this type of surgery as an access to establish cardiopulmonary bypass whether to cannulate it directly by a femoral cannula or by using synthetic tube graft. Over the last 2 decades they started to find other surgical access during this type of surgery to maintain adequate perfusion to different body organs especially the brain during cardiopulmonary bypass $(\mathrm{CPB})^{(\mathbf{1}, \mathbf{2})}$.
\end{abstract}

Background: Although many studies have reported the advantages and disadvantages between subclavian and femoral and cannulation, however most of these studies reported results for all patients underwent deep hypothermic circulatory arrest (DHCA) with ascending and aortic arch surgery with different pathologies. Objective: In our study we compared femoral and subclavian artery cannulation and reported the results only for the ascending aorta with acute type A aortic dissection (ATAAD). Patients and Methods: From January 2017 to January 2018 in Cairo University Hospitals, we selected 100 patients presented with acute type A aortic dissection and underwent reconstruction of only the ascending aorta. Patients were divided into two groups, Group (A): includes patients with subclavian artery cannulation to establish cardiopulmonary bypass (CPB) and Group (B): includes patients with femoral artery cannulation to establish CPB. Early postoperative results during hospital stay and mortality were reported and compared between the groups.

Results: The preoperative data in both groups showed no statistical difference in both groups, in postoperative data only Subclavian cannulation had a better cerebral protection and lower hospital stay than femoral cannulation $(2 \mathrm{vs} 8 \%, p=$ 0.04) while other postoperative data as well as mortality showed no statistical differences in both groups. Conclusions: From results of our study we concluded that subclavian cannulation is better than femoral cannulation in repair of type A aortic dissection as it has better cerebral protection and lower postoperative hospital stay.

Keywords: Femoral artery cannulation, Subclavian artery cannulation, Type A acute aortic dissection.

\section{PATIENTS AND METHODS}

In our study 100 patients presented by ATAAD were selected in the period between January 2017 and January 2018 in Cairo University Hospitals. Patients were divided into 2 groups; group (A): 50 patients underwent $\mathrm{CPB}$ by subclavian cannulation and group (B): 50 patients underwent CBP by femoral cannulation. All patients preoperatively were subjected to proper history taking age, sex, risk factors and past history, routine laboratory investigations, chest X-ray, echocardiography and computerized tomography (C.T) scan of chest with contrast. All patients underwent one of 3 types of surgeries; Bentall, Tirone David or supracoronary replacement of ascending aorta with resuspension of the aortic valve. All patients were assessed postoperatively by transesophageal echo (TEE).

\section{Ethical approval:}

The study was approved by the Ethics Board of Cairo University and an informed written consent was taken from each participant in the study.

Table (1): Preoperative risk factors

\begin{tabular}{|l|c|c|c|}
\hline & $\begin{array}{c}\text { Group (A) } \\
\text { N=50 }\end{array}$ & $\begin{array}{c}\text { Group (B) } \\
\text { N=50 }\end{array}$ & P value \\
\hline Age & $60 \pm 13$ & $59 \pm 11$ & NS \\
\hline Male gender & $38(76 \%)$ & $37(74 \%)$ & NS \\
\hline Hypertension & $15(30 \%)$ & $12(24 \%)$ & NS \\
\hline $\begin{array}{l}\text { Marfan } \\
\text { syndrome }\end{array}$ & $3(6 \%)$ & $2(4 \%)$ & NS \\
\hline Prenancy & $4(8 \%)$ & $2(4 \%)$ & NS \\
\hline Smoking & $22(44 \%)$ & $20(40 \%)$ & NS \\
\hline $\begin{array}{l}\text { Family history } \\
\text { of AADA }\end{array}$ & $6(12 \%)$ & $5(10 \%)$ & NS \\
\hline COPD & $11(22 \%)$ & $13(26 \%)$ & NS \\
\hline
\end{tabular}




\section{Surgical technique}

In all patients of both groups after of induction of anesthesia; right and left radial artery were cannulated and central venous pressure cannula was inserted. Routine median sternotomy was performed and pericardium was pened and then heparin was given then common atrial venous cannula was inserted in the right atrium. In group (A): left infraclavicular incision done, splitting pectoralis muscle then brachial plexus root was retracted to expose the left subclavian artery, which was closed by small $\mathrm{C}$ clamp then it was cannulated using PTFE graft size 8 french using continuous prolene 5/0 suture then the graft was connected to the arterial line after the artery declamped.

In group B: a small horizontal incision was made in the right groin to expose the femoral artery then artery was cannulated directly by a femoral cannula size 18 french by sildenger technique.

In both groups after establishing CPB, systemic cooling to $28 \mathrm{C}$ was done then artotomy was done to give intermittent cold blood cardioplegia every 30 minutes with local ice slush and aorta was cross clamped. The proximal part of the aorta with the aortic valve were done according to the technique Bentall, Davis or supracoronary replacement of the ascending aorta with hemsheild graft then systemic cooling to $24 \mathrm{OC}$ was done by asking the anesthesiologist to put the patient in Trendlenburg position and to put ice locally on the head of the patient. The superior vena cava (SVC) was selectively cannulated using right angeled venous cannula then CBP was stopped and retrograde cerebral perfusion started via SVC cannula to reconstruct the proximal part of the aorta after declamping of the aorta, then $\mathrm{CBP}$ resumed and again aorta was clamped and patient was rewarmed to 37OC. Deairing was done and aorta was declamped. Coming of bypass, reversal of heparin by protamine and proper hemostasis, TEE and closure of sternotomy were.

Patient was transferred to I.C.U on mechanical ventilation and all patients in both groups were followed up during I.C.U and hospital stay for bleeding, conscious level, stroke, mechanical ventilation time, renal and hepatic impairment, I.C.U, hospital stay and hospital mortality.

\section{Statistical methods}

Data was analyesd using the statistical package for social sciences (SPSS) version $25, \mathrm{P}$ value $<0.05$ considered statistically significant.

\section{RESULTS}

The intraoperative data are shown in table 2.

Table (2): Intraoperative data

\begin{tabular}{|l|c|c|c|}
\hline & Group (A) N=50 & Group (B) N=50 & P value \\
\hline Bentall procedure & $25(50 \%)$ & $26(52 \%)$ & Ns \\
\hline David procedure & $15(30 \%)$ & $14(28 \%)$ & Ns \\
\hline $\begin{array}{l}\text { Supracoronary conduit with } \\
\text { resuspension of the aortic valve }\end{array}$ & $10(20 \%)$ & $10(20 \%)$ & Ns \\
\hline CPB time & $185 \pm 55$ & $190 \pm 58$ & Ns \\
\hline Ischemic time & $90 \pm 33$ & $89 \pm 30$ & Ns \\
\hline Total circulatory arrest time & $31 \pm 5.7$ & $30 \pm 5.5$ & \\
\hline
\end{tabular}

Concerning the postoperative data, there was a statistically significant difference in neurological dysfunctions between both groups (Table 3).

Table (3): Postoperative data

\begin{tabular}{|c|c|c|c|}
\hline & $\begin{array}{l}\text { Group (A) } \\
\mathrm{N}=50\end{array}$ & Group $(B) N=50$ & P value \\
\hline Bleeding with reopening & $3(6 \%)$ & $4(8 \%)$ & Ns \\
\hline Renal impairment with temporary renal dialysis & $2(4 \% 0$ & $3(6 \%)$ & Ns \\
\hline Mechanical ventilation $>2$ days & $4(8 \%)$ & $5(10 \%)$ & Ns \\
\hline Pneumonia & $4(8 \%)$ & $3(6 \%)$ & Ns \\
\hline Neurological dysfunctions & $1(2 \%)$ & $4(8 \%)$ & $<0.05$ \\
\hline I.C.U stay > 4 days & $3(6 \%)$ & $4(8 \%)$ & Ns \\
\hline Hospital stay $>10$ & $3(6 \%)$ & $8(16 \%)$ & Ns \\
\hline Postoperative mortality & $2(4 \%)$ & $1(2 \%)$ & Ns \\
\hline
\end{tabular}




\section{DISCUSSION}

Most of cardiac surgeons over years used to use femoral artery as an access to establish CPB, but recently there is a trend towards use of the subclavian artery instead of femoral artery in patients with ATAAD ${ }^{(1,2,3,4,10)}$, but there is still a debate about some issues as : some surgeons consider subclavian artery cannulation is surgically much more complicated than that of femoral artery and it needs more time and of course in cases of ATAAD time is very precious as this is an emergency surgery. From point of view of many cardiac surgeons the complications of subclavian artery, if they occurred, are much more difficult to deal with unlike femoral artery.

The use of subclavian artery as an access to establish $\mathrm{CPB}$ is not commonly used in most of cardiac centers especially in ATAAD and most important of all, in some cases of the ATAAD there maybe intimal tear near the subclavian artery or even it may involve the subclavian artery, which makes it difficult to use the subclavian artery as routine to establish CPB in such cases ${ }^{(3,4,5,6)}$. In this study, only cases involving ascending aorta reconstruction was enrolled to avoid major statistical error that could have been introduced because of disease severity and procedure differences.

There are two important issues about femoral cannulation as an access to establish CPB, first is according to the Hagen-Poiseuille law ${ }^{(\mathbf{1}, \mathbf{2}, \mathbf{6})}$, the pressure drops in a fluid flowing through a long cylindrical pipe. So with continuous retrograde blood flow, cerebral perfusion may be inadequate with femoral cannulation, so injury would be higher in the femoral cannulation group. Second, it is assumed that subclavian cannulation would pump major blood flow into the true lumen, while femoral cannulation pumps more blood into the false lumen ${ }^{(3,5,7}$, 8).

Based on our evaluation, patients underwent surgery via subclavian artery had better recovery and less hospital stay than those underwent surgery via femoral cannulation as in case of subclavian artery cannulation the blood supply to different body organ went in ante grade direction unlike with femoral cannulation as the blood flow will go in retrograde direction.

Our analysis of hospital morbidity revealed major risk factor in this type of surgery which is long CPB time $>180$ min especially with total circulatory arrest. Cerebral injury has been thought to be a risk factor during major aortic surgery, particularly with retrograde continues blood flow $(1,2,9,10)$

Although the retrograde flow of femoral cannulation was eliminated as a risk factor but still there is a risk of increased morbidity and prolonged hospital stay due to neurological dysfunctions because of cerebral injury.

\section{CONCLUSION}

From results of our study we concluded that in cases of surgical repair of acute type A aortic dissection, it is better to use subclavian artery as an access for cardiopulmonary bypass than femoral artery.

\section{REFERENCES}

1. Pozrikidis C (2009): New York, London: Springer Science. Fluid Dynamics: Theory, Computation, and Numerical Simulation, 2nd edn. Pp. 362-383.

2. Girdauskas E, Kuntze T, Borger $M$ et al. (2009): Surgical risk of preoperative malperfusion in acute type A aortic dissection. $\mathbf{J}$ Thorac Cardiovasc Surg. , 138:1363-1369.

3. Wong DR, Coselli JS, Palmero L et al. (2010): Axillary artery cannulation in surgery for acute or subacute ascending aortic dissections. Ann Thorac Surg. , 90:731-737.

4. Deeb G, Patel H, Williams D (2010): Treatment for malperfusion syndrome in acute type A and B aortic dissection: a long-term analysis. J Thorac Cardiovasc Surg ;140(Suppl 6):S98-s100. Discussion,S142-S146.

5. Halkos M, Kerendi F, Myung $R$ et al. (2009): Selective antegrade cerebral perfusion via right axillary artery cannulation reduces morbidity and mortality after proximal aortic surgery. J Thorac Cardiovasc Surg.,138:1081-108.

6. Kano M, Chikugo F, Shimahara Y, Urata M, Hayamizu T (2008); Left axillary artery perfusion in surgery of type A aortic dissection. Ann Thorac Cardiovasc Surg.,14:22-4.

7. Christian D, Konstantin V, Jaqueline da $\mathrm{R}$ et al. (2014): Impact of perfusion strategy on outcome after repair for acute type A aortic dissection. Ann Thorac Surg. , 97:78-86.

8. Panos A, Murith N, Bednarkiewicz $\mathbf{M}$, Khatchatourov G (2006); Axillary cerebral perfusion for arch surgery in acute type A dissection under moderate hypothermia. Eur J Cardiothorac Surg.,29:1036-9.

9. Fong L, Bassin L, Mathur M (2013); Liberal use of axillary artery cannulation for aortic and complex cardiac surgery. Interact CardioVasc Thorac Surg.,16:755-8.

10. Hedayati N, Sherwood J, Schomisch S et al. (2004): Axillary artery cannulation for cardiopulmonary bypass reduces cerebral microemboli. J Thorac Cardiovasc Surg.,128:386-90. 